\title{
A combination of EFG-SBM and a temporally-piecewise adaptive algorithm to solve
} viscoelastic problems

\author{
X.F. Guo ${ }^{1,2}$, H.T. Yang ${ }^{1 *}$ \\ ${ }^{1}$ State Key Lab of Structural Analysis for Industrial Equipment, Dept. of Engineering Mechanics, \\ Dalian University of Technology, Dalian 116024, P.R. China \\ ${ }^{2}$ School of Civil and safety Engineering, Dalian Jiaotong University, Dalian 116028, P.R. China
}

\begin{abstract}
This paper combines Element-Free Galerkin Scaled Boundary Method (EFG-SBM) with a temporally-piecewise adaptive algorithm to solve viscoelastic problems. By expanding variables at a discretized time interval, the variations of variables can be described more precisely, and a space-time domain coupled problem can be converted into a series of recurrent boundary value problems which are solved by EFG-SBM via an adaptive computing process. Numerical tests including creep and relaxation are given to verify the proposed algorithm.
\end{abstract}

Key Words: Viscoelasticity; Element-free Galerkin scaled boundary method; temporally-piecewise adaptive algorithm.

\section{Introduction}

The Scaled Boundary Method (SBM) is a semi-analytical method originally developed by Wolf and Song in 1996 [1-2]. By introducing a normalised radial coordinate system based on a scaling centre and a defining curve (usually taken as the boundary), the governing differential equations are weakened in the circumferential direction and then solved analytically in the normalised radial direction.

As its advantages in dealing with the problems involving unbounded domains and singularity, SBM has been applied in various fields [3-9]. However there seems no any direct report relevant to its exploration on the viscoelastic problem that relates to many engineering issues [10-17]. As matter of a fact, there is a pool containing a number of spatially discretized algorithms employed for the viscoelastic analysis, such as Finite Element Method (FEM) [18-23], Boundary Element Method (BEM) [24-26] and Element-Free Galerkin Method (EFGM) [27-29], but not yet popularly used with Scaled Boundary Method.

This paper attempts to develop a SBM based numerical algorithm that may be particularly beneficial to solve viscoelastic problems with unbounded domains or singularity. On the other hand, due to the viscoelastic constitutive relationship that is time dependent, usually a step-by-step based numerical technique is required temporally. Actually there is a long list including a variety of such

\footnotetext{
${ }^{*}$ Corresponding author. State Key Lab of Structural Analysis for Industrial Equipment, Dept. of Engineering Mechanics, Dalian University of Technology, Dalian 116024, P.R. China Tel: +86-411- 84708394

E-mail address: haitian@dlut.edu.cn
} 
techniques $[23,24,27,28,30-33]$ among which a temporally-piecewised algorithm $[23,27,28]$ is one that is not only easy to combine with spatially discretized algorithms, but also able to adapt the change of the size of time step via a self-adaptive process. Therefore this paper combines advantages of Scaled Boundary Method (SBM) and a temporally-piecewise adaptive algorithm, and proposes a new approach to solve viscoelastic problems.

The paper is organized as follows, Section 1 is the introduction; Sections 2 gives recurrent viscoelastic governing equations; Section 3 presents recursive equations of EFG-SBM; Section 4 provides four numerical examples, and Section 5 goes to the conclusion.

\section{Recurrent governing equations}

The governing equations of 2-D viscoelastic problems are described by [34]

$$
\begin{array}{cr}
\text { Equilibrium equation } & \mathbf{H} \boldsymbol{\sigma}+\mathbf{b}=\mathbf{0} \\
\text { Relationship of strain and displacement } & \boldsymbol{\varepsilon}=\mathbf{H}^{\mathrm{T}} \mathbf{u}
\end{array}
$$

where $\boldsymbol{\sigma}$ and $\boldsymbol{\varepsilon}$ denote the vector of stress and strain, respectively, $\mathbf{b}$ is the vector of body force, $\mathbf{u}$ is the vector of displacement,

$$
\mathbf{H}=\left[\begin{array}{ccc}
\partial / \partial x & 0 & \partial / \partial y \\
0 & \partial / \partial y & \partial / \partial x
\end{array}\right]
$$

The viscoelastic constitutive relationships is specified by a three-parameter solid viscoelasticity model (see Fig.1) in a differential form [10-12]

$$
\begin{gathered}
\alpha_{1} \mathbf{D} \boldsymbol{\varepsilon}(t)+p_{1} \mathbf{D} \frac{\mathrm{d} \boldsymbol{\varepsilon}(t)}{\mathrm{d} t}=\left(\alpha_{1}+\alpha_{2}\right) \boldsymbol{\sigma}(t)+p_{1} \frac{\mathrm{d} \boldsymbol{\sigma}(t)}{\mathrm{d} t} \quad(t>0) \\
\boldsymbol{\sigma}(t)=\mathbf{D} \boldsymbol{\varepsilon}(t) \quad(t=0
\end{gathered}
$$

where

$$
\begin{gathered}
\mathbf{D}=\frac{E_{2}}{1-v^{2}}\left(\begin{array}{ccc}
1 & v & 0 \\
v & 1 & 0 \\
0 & 0 & \frac{1-v}{2}
\end{array}\right) \quad \text { for the plane stress probl } \\
p_{1}=\frac{\eta_{1}}{E_{1}+E_{2}} \quad \alpha_{1}=\frac{E_{1}}{E_{1}+E_{2}} \quad \alpha_{2}=\frac{E_{2}}{E_{1}+E_{2}}
\end{gathered}
$$

where $v, E_{1}, E_{2}$ and $\eta_{1}$ are constitutive parameters.

For the plane strain problems, $E_{1}, E_{2}, \eta_{1}$ and $v$ need to be replaced by $\frac{E_{1}}{1-v^{2}}, \frac{E_{2}}{1-v^{2}}$, $\frac{\eta_{1}}{1-v^{2}}$, and $\frac{v}{1-v}$, respectively.

The boundary conditions are given by [34]

$$
\mathbf{u}=\tilde{\mathbf{u}} \quad \text { on } \quad \Gamma_{u}
$$




$$
\mathbf{p}=\tilde{\mathbf{p}} \quad \text { on } \quad \Gamma_{\sigma}
$$

where $\mathbf{p}$ denotes the vector of traction, $\tilde{\mathbf{u}}, \tilde{\mathbf{p}}$ are the prescribed functions on the boundary.

Divide time domain into a number of time intervals, the initial points and sizes of the time intervals are defined by $t_{0}, t_{1}, t_{2}, \ldots, t_{k} \ldots$ and $T_{1}, T_{2}, \ldots, T_{k} \ldots$, respectively. At the $k$-th discretized time interval, in order to describe the variation of variables more precisely, all variables are expanded in terms of $s_{t}$

$$
\begin{gathered}
\boldsymbol{\sigma}=\sum_{m=0} \boldsymbol{\sigma}^{k m} s_{t}^{m} \\
\boldsymbol{\varepsilon}=\sum_{m=0} \boldsymbol{\varepsilon}^{k m} s_{t}^{m} \\
\mathbf{b}=\sum_{m=0} \mathbf{b}^{k m} s_{t}^{m} \\
\mathbf{u}=\sum_{m=0} \mathbf{u}^{k m} s_{t}^{m} \\
\tilde{\mathbf{u}}=\sum_{m=0} \tilde{\mathbf{u}}^{k m} s_{t}^{m} \\
\mathbf{p}=\sum_{m=0} \mathbf{p}^{k m} s_{t}^{m} \\
\tilde{\mathbf{p}}=\sum_{m=0} \tilde{\mathbf{p}}^{k m} s_{t}^{m} \\
s_{t}=\frac{t-t_{k-1}}{T_{k}}
\end{gathered}
$$

where $t_{k-1}$ and $T_{k}$ represent the initial point and size of the $k$-th time interval, respectively, $\boldsymbol{\sigma}^{k m}$ and $\boldsymbol{\varepsilon}^{k m}$ represent the expanding coefficients of $\boldsymbol{\sigma}$ and $\boldsymbol{\varepsilon}$ at $k$-th time interval, respectively, $m$ stands for the power order of expansion, $\mathbf{b}^{k m}$ denotes the expanding coefficient of $\mathbf{b}, \mathbf{u}^{k m}, \mathbf{p}^{k m}, \tilde{\mathbf{u}}^{k m}$ and $\tilde{\mathbf{p}}^{k m}$ are the expanding coefficients of $\mathbf{u}, \mathbf{p}, \tilde{\mathbf{u}}$ and $\tilde{\mathbf{p}}, k$-th time interval, respectively.

The conversion relationship between the differentiations respect to $t$ and $s_{t}$ is

$$
\frac{\mathrm{d}}{\mathrm{d} t}=\frac{1}{T_{k}} \frac{\mathrm{d}}{\mathrm{d} s_{t}}
$$

Substituting Eqns (10-16) into Eqns $(1,2,5,8,9)$ then yields

$$
\begin{array}{rlrl}
\mathbf{H} \boldsymbol{\sigma}^{k m}+\mathbf{b}^{k m}=\mathbf{0} & \\
\boldsymbol{\varepsilon}^{k m}=\mathbf{H}^{\mathrm{T}} \mathbf{u}^{k m} & & \\
\mathbf{u}^{k m}=\tilde{\mathbf{u}}^{k m} & \text { on } & \Gamma_{u} \\
\mathbf{p}^{k m} & =\tilde{\mathbf{p}}^{k m} & \text { on } & \Gamma_{\sigma}
\end{array}
$$

At the first time interval $(k=1)$, when $m=0$ i.e $t=0$,

$$
\boldsymbol{\sigma}^{k m}=\boldsymbol{\sigma}^{10}=\mathbf{D} \boldsymbol{\varepsilon}^{k m}=\mathbf{D} \boldsymbol{\varepsilon}^{10}
$$


$\boldsymbol{\sigma}^{10}, \boldsymbol{\varepsilon}^{10}$, and $\mathbf{u}^{10}$ can be obtained by solving Eqns (19-23)

At other time intervals $(k=2,3, \ldots)$

$$
\begin{aligned}
\boldsymbol{\varepsilon}^{k 0} & =\sum_{m=0} \boldsymbol{\varepsilon}^{(k-1) m} \\
\boldsymbol{\sigma}^{k 0} & =\sum_{m=0} \boldsymbol{\sigma}^{(k-1) m} \\
\mathbf{u}^{k 0} & =\sum_{m=0} \mathbf{u}^{(k-1) m}
\end{aligned}
$$

For $m \neq 0$, substitution Eqns (10-11) for Eqn (4) then yields

$$
\begin{gathered}
\boldsymbol{\sigma}^{k m}=\mathbf{D} \boldsymbol{\varepsilon}^{k m}+\mathbf{C}^{k m} \quad k=1,2,3, \ldots \quad m=1,2, \ldots \\
\mathbf{C}^{k m}=\frac{T_{k} E_{1}}{m \eta_{1}} \mathbf{D} \boldsymbol{\varepsilon}^{k(m-1)}-\frac{T_{k}}{m p_{1}} \boldsymbol{\sigma}^{k(m-1)}
\end{gathered}
$$

Futhermore

$$
\boldsymbol{\sigma}^{k m}=\mathbf{D} \boldsymbol{\varepsilon}^{k m}+\beta_{m}\left(\alpha_{2} \mathbf{D} \sum_{i=1}^{m-1} \gamma_{i} \boldsymbol{\varepsilon}^{k i}-\alpha_{1} \mathbf{D} \boldsymbol{\varepsilon}^{k 0}+\boldsymbol{\sigma}^{k 0}\right)
$$

where

$$
\begin{aligned}
& \beta_{m}=\frac{1}{m !}\left(\frac{-T_{k}}{p_{1}}\right)^{(m)} \\
& \gamma_{i}=i !\left(\frac{-p_{1}}{T_{k}}\right)^{i}
\end{aligned}
$$

\section{Recursive equations of EFG-SBM}

The scaled boundary method introduces such a $\xi$-s coordinate system by scaling a defining curve relative to a scaling centre $\left(x_{0}, y_{0}\right)$ selected within the domain, as shown in Fig. 2 . The coordinate $\xi$ runs from the scaling centre towards the curve, and has values of zero at the scaling centre and unity at the curve. The coordinate $s$ specifies a distance around the boundary from an origin on the boundary [6-9].

The scaled and Cartesian coordinate systems are related by Deeks and Wolf [6-9]

$$
\begin{aligned}
& x=x_{0}+\xi x_{s}(s) \\
& y=y_{0}+\xi y_{s}(s)
\end{aligned}
$$

For Eqns (15-18)

$$
\mathbf{u}^{k m}(\xi, s)=\mathbf{N}(s) \mathbf{u}_{h}^{k m}(\xi)
$$

where $\mathbf{u}^{k m}(\xi, s)$ represents the expanding coefficient of $\mathbf{u}=\sum_{m=0} \mathbf{u}^{k m} s_{t}^{m}, \mathbf{N}(s)$ is a matrix of shape function and $\mathbf{u}_{h}^{k m}(\xi)$ is a set of $n$ functions analytical in $\xi$.

The expanding coefficient of the strain $\boldsymbol{\varepsilon}=\sum_{m=0} \boldsymbol{\varepsilon}^{k m} s_{t}^{m}$ in the scaled coordinate system is described 
by [6-9]

$$
\begin{gathered}
\boldsymbol{\varepsilon}^{k m}(\xi, s)=\mathbf{B}_{1}(s) \mathbf{u}^{k m}(\xi)_{, \xi}+\frac{1}{\xi} \mathbf{B}_{2}(s) \mathbf{u}^{k m}(\xi) \\
\mathbf{B}_{1}(s)=\mathbf{b}_{1}(s) \mathbf{N}(s)=\frac{1}{|\mathbf{J}|}\left[\begin{array}{ccc}
\frac{\partial y_{s}(s)}{\partial s} & 0 & -\frac{\partial x_{s}(s)}{\partial s} \\
0 & -\frac{\partial x_{s}(s)}{\partial s} & \frac{\partial y_{s}(s)}{\partial s}
\end{array}\right]^{\mathrm{T}} \cdot \mathbf{N}(s) \\
\mathbf{B}_{2}(s)=\mathbf{b}_{2}(s) \mathbf{N}(s)_{, s}=\frac{1}{|\mathbf{J}|}\left[\begin{array}{ccc}
-y_{s}(s) & 0 & x_{s}(s) \\
0 & x_{s}(s) & -y_{s}(s)
\end{array}\right]^{\mathrm{T}} \cdot \mathbf{N}(s)_{, s} \\
|\mathbf{J}|=x_{s}(s) y_{s}(s)_{, s}-y_{s}(s) x_{s}(s)_{, s}
\end{gathered}
$$

where $\mathbf{N}(s){ }_{s}$ refers to the derivative of $\mathbf{N}(s)$ with respect to $s$.

Utilizing the virtual displacement principle then gives (in the absence of body force) [6-9]

$$
\begin{gathered}
\int_{V} \delta \boldsymbol{\varepsilon}(\xi, s)^{\mathrm{T}} \boldsymbol{\sigma}^{k m}(\xi, s) \mathrm{d} V-\int_{S} \delta \mathbf{u}(s)^{\mathrm{T}} \mathbf{p}^{k m}(s) \mathrm{d} s=0 \\
m=0,1,2,3, \ldots k=1,2,3, \ldots
\end{gathered}
$$

where $\delta \mathbf{u}(\xi, s)=\mathbf{N}(s) \delta \mathbf{u}(\xi)$ denotes the vectour of virtual displacement, and

$$
\delta \boldsymbol{\varepsilon}(\xi, s)=\mathbf{B}_{1}(s) \delta \mathbf{u}(\xi)_{, \xi}+\frac{1}{\xi} \mathbf{B}_{2}(s) \delta \mathbf{u}(\xi)
$$

The second term in Eqn (38) becomes

$$
\int_{S} \delta \mathbf{u}(s)^{\mathrm{T}} \mathbf{p}^{k m}(s) \mathrm{d} s=\delta \mathbf{u}^{\mathrm{T}} \int_{S} \mathbf{N}(s)^{\mathrm{T}} \mathbf{p}^{k m}(s) \mathrm{d} s=\delta \mathbf{u}^{\mathrm{T}} \mathbf{P}^{k m} \quad k=1,2, \ldots m=0,1,2, \ldots
$$

At the first time interval, when $k=1$ and $m=0$, i.e. $t=0$, substituting Eqn (23) into Eqn (38) then gives

$$
\int_{V} \delta \boldsymbol{\varepsilon}(\xi, s)^{\mathrm{T}} \mathbf{D} \boldsymbol{\varepsilon}^{k 0}(\xi, s) \mathrm{d} V-\int_{S} \delta \mathbf{u}(s)^{\mathrm{T}} \mathbf{p}^{k 0}(s) \mathrm{d} s=0
$$

Using Eqns (34), (39-40) and Green's Theorem then gives [6-9]

$$
\begin{aligned}
\delta \mathbf{u}^{\mathrm{T}} & \left\{\mathbf{E}_{0} \mathbf{u}_{h, \xi}^{k 0}+\mathbf{E}_{1}^{\mathrm{T}} \mathbf{u}_{h}^{k 0}\right\}-\delta \mathbf{u}^{\mathrm{T}} \int_{S} \mathbf{N}(s)^{\mathrm{T}} \mathbf{p}^{k 0}(s) \mathrm{d} s \\
& -\int_{0}^{1} \delta \mathbf{u}(\xi)^{\mathrm{T}}\left\{\mathbf{E}_{0} \xi \mathbf{u}_{h}^{k 0}(\xi)_{, \xi \xi}+\left[\mathbf{E}_{0}+\mathbf{E}_{1}^{\mathrm{T}}-\mathbf{E}_{1}\right] \mathbf{u}_{h}^{k 0}(\xi)_{, \xi}-\mathbf{E}_{2} \frac{1}{\xi} \mathbf{u}_{h}^{k 0}(\xi)\right\} \mathrm{d} \xi=0
\end{aligned}
$$

where

$$
\begin{aligned}
\mathbf{E}_{0} & =\int_{S} \mathbf{B}_{1}(\mathrm{~s})^{\mathrm{T}} \mathbf{D} \mathbf{B}_{1}(\mathrm{~s})|\mathbf{J}(s)| \mathrm{d} s \\
\mathbf{E}_{1} & =\int_{S} \mathbf{B}_{2}(\mathrm{~s})^{\mathrm{T}} \mathbf{D} \mathbf{B}_{1}(\mathrm{~s})|\mathbf{J}(s)| \mathrm{d} s
\end{aligned}
$$




$$
\mathbf{E}_{2}=\int_{S} \mathbf{B}_{2}(\mathrm{~s})^{\mathrm{T}} \mathbf{D} \mathbf{B}_{2}(\mathrm{~s})|\mathbf{J}(s)| \mathrm{d} s
$$

Furthermore

$$
\begin{gathered}
\mathbf{P}^{* k 0}=\mathbf{E}_{0} \mathbf{u}_{h, \xi}^{k 0}+\mathbf{E}_{1}^{\mathrm{T}} \mathbf{u}_{h}^{k 0} \\
\mathbf{E}_{0} \xi^{2} \mathbf{u}_{h}^{k 0}(\xi)_{, \xi \xi}+\left[\mathbf{E}_{0}+\mathbf{E}_{1}^{\mathrm{T}}-\mathbf{E}_{1}\right] \xi \mathbf{u}_{h}^{k 0}(\xi)_{, \xi}-\mathbf{E}_{2} \mathbf{u}_{h}^{k 0}(\xi)=\mathbf{0}
\end{gathered}
$$

where

$$
\mathbf{P}^{* k 0}=\mathbf{P}^{k 0}=\int_{S} \mathbf{N}(s)^{\mathrm{T}} \mathbf{p}^{k 0}(s) \mathrm{d} s
$$

In Eqns (42), (44) and the following section, $\mathbf{u}_{h, \xi}^{k m}$ and $\mathbf{u}_{h}^{k m}$ are used to represent $\mathbf{u}_{h, \xi}^{k m}(\xi=1)$ and $\mathbf{u}_{h}^{k m}(\xi=1)$.

When $t>0$, substituting Eqn (29) into Eqn (38) then gives

$$
\begin{array}{r}
\int_{V} \delta \boldsymbol{\varepsilon}(\xi, s)^{\mathrm{T}} \mathbf{D} \boldsymbol{\varepsilon}^{k m} \mathrm{~d} V+\beta_{m} \alpha_{2} \sum_{i=1}^{m-1} \gamma_{i} \int_{V} \delta \boldsymbol{\varepsilon}(\xi, s)^{\mathrm{T}} \mathbf{D} \boldsymbol{\varepsilon}^{k i} \mathrm{~d} V-\beta_{m} \alpha_{1} \int_{V} \delta \boldsymbol{\varepsilon}(\xi, s)^{\mathrm{T}} \mathbf{D} \boldsymbol{\varepsilon}^{k 0} \mathrm{~d} V \\
+\beta_{m} \int_{V} \delta \boldsymbol{\varepsilon}(\xi, s)^{\mathrm{T}} \boldsymbol{\sigma}^{k 0} \mathrm{~d} V-\int_{S} \delta \mathbf{u}(s)^{\mathrm{T}} \mathbf{p}^{k m}(s) \mathrm{d} s=0
\end{array}
$$

Using Eqns (34), (38-40) and Green's Theorem then yields [6-7]

$$
\begin{aligned}
& \delta \mathbf{u}^{\mathrm{T}}\left\{\mathbf{E}_{0} \mathbf{u}_{h, \xi}^{k m}+\mathbf{E}_{1}^{\mathrm{T}} \mathbf{u}_{h}^{k m}\right\} \\
& +\delta \mathbf{u}^{\mathrm{T}}\left\{\beta_{m} \alpha_{2} \sum_{i=1}^{m-1} \gamma_{i}\left\{\mathbf{E}_{0} \mathbf{u}_{h, \xi}^{k i}+\mathbf{E}_{1}^{\mathrm{T}} \mathbf{u}_{h}^{k i}\right\}-\beta_{m} \alpha_{1}\left\{\mathbf{E}_{0} \mathbf{u}_{h, \xi}^{k 0}+\mathbf{E}_{1}^{\mathrm{T}} \mathbf{u}_{h}^{k 0}\right\}+\beta_{m} \mathbf{P}^{k 0}-\mathbf{P}^{k m}\right\} \\
& -\beta_{m} \alpha_{2} \sum_{i=1}^{m-1} \gamma_{i} \int_{0}^{1} \delta \mathbf{u}(\xi)^{\mathrm{T}}\left\{\mathbf{E}_{0} \xi \mathbf{u}_{h}^{k i}(\xi)_{, \xi \xi}+\left[\mathbf{E}_{0}+\mathbf{E}_{1}^{\mathrm{T}}-\mathbf{E}_{1}\right] \mathbf{u}_{h}^{k i}(\xi)_{, \xi}-\mathbf{E}_{2} \frac{1}{\xi} \mathbf{u}_{h}^{k i}(\xi)\right\} \mathrm{d} \xi \\
& +\beta_{m} \alpha_{1} \int_{0}^{1} \delta \mathbf{u}(\xi)^{\mathrm{T}}\left\{\mathbf{E}_{0} \xi \mathbf{u}_{h}^{k 0}(\xi)_{, \xi \xi}+\left[\mathbf{E}_{0}+\mathbf{E}_{1}^{\mathrm{T}}-\mathbf{E}_{1}\right] \mathbf{u}_{h}^{k 0}(\xi)_{, \xi}-\mathbf{E}_{2} \frac{1}{\xi} \mathbf{u}_{h}^{k 0}(\xi)\right\} \mathrm{d} \xi \\
& -\int_{0}^{1} \delta \mathbf{u}(\xi)^{\mathrm{T}}\left\{\mathbf{E}_{0} \xi \mathbf{u}_{h}^{k m}(\xi)_{, \xi \xi}+\left[\mathbf{E}_{0}+\mathbf{E}_{1}^{\mathrm{T}}-\mathbf{E}_{1}\right] \mathbf{u}_{h}^{k m}(\xi)_{, \xi}-\mathbf{E}_{2} \frac{1}{\xi} \mathbf{u}_{h}^{k m}(\xi)\right\} \mathrm{d} \xi=\mathbf{0}
\end{aligned}
$$

where

$$
\mathbf{P}^{k 0}=\int_{S} \mathbf{N}(s)^{\mathrm{T}} \mathbf{p}^{k 0}(s) \mathrm{d} s=\sum_{m=0} \mathbf{P}^{(k-1) m} \quad k=2,3, \ldots
$$

Furthermore

$$
\begin{gathered}
\mathbf{P}^{* k m}=\mathbf{E}_{0} \mathbf{u}_{h, \xi}^{k m}+\mathbf{E}_{1}^{\mathrm{T}} \mathbf{u}_{h}^{k m} \\
\mathbf{E}_{0} \xi^{2} \mathbf{u}_{h}^{k i}(\xi)_{, \xi \xi}+\left[\mathbf{E}_{0}+\mathbf{E}_{1}^{\mathrm{T}}-\mathbf{E}_{1}\right] \xi \mathbf{u}_{h}^{k i}(\xi)_{, \xi}-\mathbf{E}_{2} \mathbf{u}_{h}^{k i}(\xi)=\mathbf{0} \quad i=0,1,2, \ldots m
\end{gathered}
$$

where

$$
\mathbf{P}^{* k m}=\mathbf{P}^{k m}-\beta_{m} \alpha_{2} \sum_{i=1}^{m-1} \gamma_{i} \mathbf{P}^{* k i}+\beta_{m} \alpha_{1} \mathbf{P}^{* k 0}-\beta_{m} \mathbf{P}^{k 0} \quad m=1,2, \ldots
$$




$$
\mathbf{P}^{* k i}=\mathbf{E}_{0} \mathbf{u}_{h, \xi}^{k i}+\mathbf{E}_{1}^{\mathrm{T}} \mathbf{u}_{h}^{k i} \quad i=0,1,2, \ldots, m-1
$$

$\mathbf{u}^{k m}(\xi)$ is approximated by

$$
\mathbf{u}^{k m}(\xi)=c_{1}^{k m} \xi^{-\lambda_{1}} \phi_{1}+c_{2}^{k m} \xi^{-\lambda_{2}} \phi_{2}+\ldots=\sum_{i=1}^{n} c_{i}^{k m} \xi^{-\lambda_{i}} \phi_{i} \quad k=1,2, \ldots m=0,1,2, \ldots
$$

When $\xi=1$

$$
\mathbf{u}_{h}^{k m}=c_{1}^{k m} \boldsymbol{\phi}_{1}+c_{2}^{k m} \boldsymbol{\phi}_{2}+\ldots=\sum_{i=1}^{n} c_{i}^{k m} \boldsymbol{\phi}_{i}=\boldsymbol{\Phi} \mathbf{c}^{k m}
$$

where

$$
\begin{aligned}
\boldsymbol{\Phi} & =\left[\begin{array}{llll}
\boldsymbol{\phi}_{1} & \boldsymbol{\phi}_{2} & \cdots & \boldsymbol{\phi}_{n}
\end{array}\right] \\
\mathbf{c}^{k m} & =\left[c_{1}^{k m}, c_{2}^{k m}, \ldots, c_{n}^{k m}\right]^{\mathrm{T}}
\end{aligned}
$$

Therefore

$$
\begin{aligned}
{\left[\lambda_{i}^{2} \mathbf{E}_{0}-\lambda_{i}\right.} & {\left.\left[\mathbf{E}_{1}^{\mathrm{T}}-\mathbf{E}_{1}\right]-\mathbf{E}_{2}\right] \boldsymbol{\phi}_{i}=\mathbf{0} } \\
\mathbf{P}^{* k m} & =\mathbf{Q} \mathbf{c}^{k m}
\end{aligned}
$$

where

$$
\begin{aligned}
\mathbf{Q} & =\left[\begin{array}{llll}
\mathbf{q}_{1} & \mathbf{q}_{2} & \cdots & \mathbf{q}_{n}
\end{array}\right] \\
\mathbf{q}_{i} & =\left[\begin{array}{ll}
\mathbf{E}_{1}^{\mathrm{T}}-\lambda_{i} \mathbf{E}_{0} & \boldsymbol{\phi}_{i}
\end{array}\right.
\end{aligned}
$$

Eqns (58) and (61) can be further expressed by [6-9]

$$
\left[\begin{array}{cc}
\mathbf{E}_{\mathbf{0}}^{-1} \mathbf{E}_{1}^{\mathrm{T}} & -\mathbf{E}_{\mathbf{0}}^{-1} \\
\mathbf{E}_{1} \mathbf{E}_{0}^{-1} \mathbf{E}_{1}^{\mathrm{T}}-\mathbf{E}_{2} & -\mathbf{E}_{1} \mathbf{E}_{0}^{-1}
\end{array}\right]\left\{\begin{array}{c}
\boldsymbol{\phi} \\
\mathbf{q}
\end{array}\right\}=\lambda\left\{\begin{array}{c}
\boldsymbol{\phi} \\
\mathbf{q}
\end{array}\right\}
$$

Left-multiplying both sides of Eqn (55) with $\boldsymbol{\Phi}^{-1}$ then yields

$$
\mathbf{c}^{k m}=\boldsymbol{\Phi}^{-1} \mathbf{u}_{h}^{k m}
$$

Thus

$$
\mathbf{Q} \mathbf{c}^{k m}=\mathbf{Q} \Phi^{-1} \mathbf{u}_{h}^{k m}=\mathbf{K} \mathbf{u}_{h}^{k m}=\mathbf{P}^{* k m} \quad k=1,2, \ldots m=0,1,2, \ldots
$$

The boundary $\xi=1$ is divided into several segments at each of which $N(s)$ is defined by EFGM [7-9, 35, 36].

$$
\left[N_{1}(s), \ldots, N_{n_{l}}(s)\right]=\varphi(s)^{\mathrm{T}} \Theta^{-1}
$$

where

$$
\boldsymbol{\varphi}(s)^{\mathrm{T}}=\left[\varphi_{1}(s) \varphi_{2}(s) \ldots \varphi_{n_{l}}(s)\right]
$$


$n_{l}$ is the total number of nodes at the $l$ th segment. $n_{x}$ is the total number of nodes in the domain of definition of point $s$ at the segment.

A spline weight function [7-9] is employed in this paper, i.e.

$$
w_{I}(s)= \begin{cases}1-6\left(\frac{\left|s-s_{I}\right|}{r_{I}}\right)^{2}+8\left(\frac{\left|s-s_{I}\right|}{r_{I}}\right)^{3}-3\left(\frac{\left|s-s_{I}\right|}{r_{I}}\right)^{4} & 0<\left|s-s_{I}\right|<r_{I} \\ 0 & \left|s-s_{I}\right| \geq r_{I}\end{cases}
$$

where $s_{I}$ is $s$ coordinate of $\quad I$ th node, and $r_{I}$ is the size of support domain.

Therefore

$$
\left[N_{1}(s)_{, s}, \ldots, N_{n_{l}}(s)_{, s}\right]=\boldsymbol{\varphi}(s)_{, s}^{\mathrm{T}} \boldsymbol{\Theta}^{-1}
$$

where

$$
\begin{gathered}
\boldsymbol{\varphi}(s)_{, s}^{\mathrm{T}}=\mathbf{p}(s)_{, s}^{\mathrm{T}} \mathbf{A}(s)^{-1} \mathbf{H}(s)+\mathbf{p}(s)^{\mathrm{T}}\left[\mathbf{A}(s)_{, s}^{-1} \mathbf{H}(s)+\mathbf{A}(s)^{-1} \mathbf{H}(s)_{, s}\right] \\
\mathbf{A}(s)_{, s}^{-1}=-\mathbf{A}(s)^{-1} \mathbf{A}(s)_{, s} \mathbf{A}(s)^{-1}
\end{gathered}
$$

Using Eqn (64) in which stiffness matrix $\mathbf{K}$ needs to be generated only one time, the solution of a viscoelastic problem can be achieved in a recursive process, at the beginning of each time interval, $\mathbf{u}^{k 0}$ is determined by solving an elastic problem defined via Eqn (23) or by Eqn (26).

A self-adaptive computation is carried out at each of the time intervals with a convergence criterion

$$
\operatorname{Abs}\left(\left(u_{h j}^{k R} s_{t}^{R} / \sum_{m=0}^{R-1} u_{h j}^{k m} s_{t}^{m}\right)_{s_{t}=1}\right) \leq \beta
$$

where $\beta$ is an error bound, $u_{h j}^{k m}$ denotes the $j$-th component of $\mathbf{u}_{h}^{k m}(m=1,2, \ldots, R)$. Every $\mathbf{u}_{h}^{k R}(R$ $=1,2, \ldots)$ is required to be checked with the above criterion, if the criterion is satisfied consecutively 3 times, computing will stop at the time interval considered, and step into the next one. If the criterion is not met, the next order $(R+1)$ computation will continue till reaching the convergence.

In the computation, $m m$ and mim, the upper and lower bound of $R$, will be prescribed in advance. 
If condition (76) is not satisfied when $R=m m$, a size decrement of time step is necessary to restart the recursive procedure at the current time interval; if condition (76) is satisfied when $R<\operatorname{mim}$, a size increment of the time step can be considered at the next time interval.

It seems difficult to give a rigorous way to determine $\mathrm{mm}, \mathrm{mim}$, and sizes increment and decrement of time steps, in this paper, $m m=20, \operatorname{mim}=5$, size increment and decrement are both half size of current time step for the convenience.

Eqn (64) can be rearranged as

$$
\left[\begin{array}{ll}
\mathbf{K}_{a a} & \mathbf{K}_{a b} \\
\mathbf{K}_{b a} & \mathbf{K}_{b b}
\end{array}\right]\left\{\begin{array}{l}
\mathbf{u}_{h a}^{k m} \\
\overline{\mathbf{u}}_{h b}^{k m}
\end{array}\right\}=\left\{\begin{array}{l}
\overline{\mathbf{P}}_{a}^{* k m} \\
\mathbf{P}_{b}^{* k m}
\end{array}\right\} \quad k=1,2, \ldots m=0,1,2, \ldots
$$

where $\mathbf{u}_{h a}^{k m}$ and $\overline{\mathbf{u}}_{h b}^{k m}$ represent the unknown and known expanding coefficients of nodal displacements, respectively, while $\overline{\mathbf{P}}_{a}^{* k m}$ and $\mathbf{P}_{b}^{* k m}$ represent the corresponding known and unknown expanding coefficients of nodal forces, respectively.

Furthermore, the following equations can be obtained

$$
\begin{aligned}
\mathbf{K}_{a a} \mathbf{u}_{h a}^{k m}+\mathbf{K}_{a b} \overline{\mathbf{u}}_{h b}^{k m}=\overline{\mathbf{P}}_{a}^{* k m} \\
\mathbf{K}_{b a} \mathbf{u}_{h a}^{k m}+\mathbf{K}_{b b} \overline{\mathbf{u}}_{h b}^{k m}=\mathbf{P}_{b}^{* k m}
\end{aligned}
$$

Via Eqn (78), the expanding coefficient of unknown nodal displacements can be obtained as follow

$$
\mathbf{u}_{h a}^{k m}=\mathbf{K}_{a a}^{-1}\left(\overline{\mathbf{P}}_{a}^{* k m}-\mathbf{K}_{a b} \overline{\mathbf{u}}_{h b}^{k m}\right) \quad k=1,2, \ldots m=0,1,2, \ldots
$$

For a special case where

$$
\begin{array}{rlrl}
\overline{\mathbf{u}}_{h b}^{k 0} & =\overline{\mathbf{u}}_{h b}^{(k-1) 0} & k=2,3, \ldots m=0 \\
\overline{\mathbf{u}}_{h b}^{k m} & =\mathbf{0} & k=1,2, \ldots m=1,2, \ldots \\
\overline{\mathbf{P}}_{a}^{k m} & =\mathbf{0} & k=1,2, \ldots m=0,1,2, \ldots
\end{array}
$$

It can be proved

$$
\begin{array}{ll}
\overline{\mathbf{P}}_{a}^{* k m}=\mathbf{0} & k=1,2, \ldots m=0,1,2, \ldots \\
\mathbf{u}_{h a}^{k m}=\mathbf{0} & k=1,2, \ldots m=1,2, \ldots
\end{array}
$$

Furthermore

$$
\begin{array}{ccc}
\mathbf{u}_{h}^{k m}=\left\{\begin{array}{l}
\mathbf{u}_{h a}^{k m} \\
\overline{\mathbf{u}}_{h b}^{k m}
\end{array}\right\}=\mathbf{0} & k=1,2, \ldots m=1,2, \ldots \\
\mathbf{u}_{h}^{k 0}=\left\{\begin{array}{l}
\mathbf{u}_{h a}^{k 0} \\
\overline{\mathbf{u}}_{h b}^{k 0}
\end{array}\right\}=\left\{\begin{array}{l}
\mathbf{u}_{h a}^{(k-1) 0} \\
\overline{\mathbf{u}}_{h b}^{(k-1) 0}
\end{array}\right\}=\mathbf{u}_{h}^{(k-1) 0} & k=2,3, \ldots m=0 \\
\boldsymbol{\varepsilon}^{k m}=\mathbf{0} & k=1,2, \ldots m=1,2, \ldots \\
\boldsymbol{\varepsilon}^{k 0}=\boldsymbol{\varepsilon}^{(k-1) 0} & k=2,3, \ldots m=0 \\
\boldsymbol{\sigma}^{k m}=\beta_{m}\left(-\alpha_{1} \boldsymbol{\sigma}^{j 0}+\boldsymbol{\sigma}^{k 0}\right) & k=1,2, \ldots m=1,2, \ldots j=1
\end{array}
$$




\section{Numerical examples}

Four numerical examples including creep and relaxation analysis are given to verify the proposed algorithm.

For the creep analysis, we assume $\mathbf{b}=\mathbf{0} \tilde{\mathbf{u}}=0, \tilde{\mathbf{p}}$ is time independent; for the relaxation analysis we assume $\mathbf{b}=\mathbf{0} \tilde{\mathbf{p}}=0, \tilde{\mathbf{u}}$ is time independent.

Under the above assumptions, it can be proved that the solution of displacement in a creep problem and the solution of stress in a relaxation problem can be presented using a product of creep compliance with an elastic displacement solution or a product of relaxation modulus with an elastic stress solution. The elastic solutions can be obtained by either analytical means or ANSYS [11]. In this way, the reference solutions can be gained, and used for the comparison with the solutions of proposed algorithm.

For all examples, $\beta=1 \times 10^{-9}$.

\section{Numerical Example 1}

Consider a square viscoelastic plate. Regarding to the creep and relaxation cases, two boundary conditions are given in Fig. 3 (a) and Fig. 3 (b) where $l=1 \mathrm{~m}, q=1 \mathrm{~N} / \mathrm{m}$, and $u_{x}=5 \mathrm{~mm}$. Plane stress conditions are assumed with $E_{1}=0.5 \mathrm{MPa}, E_{2}=1 \mathrm{MPa}, \eta_{1}=1 \times 10^{7} \mathrm{~N} \cdot \mathrm{s} / \mathrm{m}^{2}$ and $v=0.25$. Nodes are uniformly arranged along the boundary with a uniform $\Delta \mathrm{s}=0.25 \mathrm{~m}$.

The solution of $u_{x}$ at node $A$ given by the proposed algorithm with uniform sizes of time steps (10 seconds) is presented in Table 1 and Fig.4, and is compared with a reference solution.

The solution of $\sigma_{x x}$ at node $B$ given by the proposed algorithm with uniform sizes of time steps (10 seconds) is presented in Table 2 and Fig.5, and is compared with a reference solution [37].

\section{Numerical Example 2}

Consider a square plate with a square hole. Regarding to creep and relaxation cases, two boundary conditions are given in Fig. 6 (a) and Fig. 6 (b) where $a=1 \mathrm{~m}, q=1 \mathrm{~N} / \mathrm{m}$ and $u_{x}=5 \mathrm{~cm}$. Due to the biaxial symmetry, only one quarter of the plate is taken into account as shown in Fig. 6 (c) and Fig. 6 (d). Plane stress conditions are assumed with $E_{1}=2000 \mathrm{~Pa}, E_{2}=2000 \mathrm{~Pa}, \eta_{1}=8 \times 10^{5} \mathrm{~N} \cdot \mathrm{s} / \mathrm{m}^{2}$ and $v=0.3$.

Nodes are arranged along the boundary with a uniform $\Delta \mathrm{s}=0.25 \mathrm{~m}$, except side faces.

The results obtained by the proposed approach is compared with a reference solution whose elastic solution is given by ANSYS with 15309 nodes and 7362 6-node-triangular finite elements.

The solution of $u_{y}$ at point $O$ given by the proposed algorithm with adaptive sizes of time steps is presented in Table 3 where 1 check, 2 checks and 3 checks mean that the criterion (76) is satisfied only 1 time, consecutively 2 and 3 times respectively. The maximum relative error between the proposed algorithm and reference solution is about 0.03\%. Fig. 8 and Fig. 9 exhibit the adaptive procedures of size of time interval and power order, respectively.

The solution of $\sigma_{x x}$ at node $H$ given by the proposed algorithm with adaptive sizes of time steps is presented in Table 4 and Fig. 10, and The maximum relative error between the proposed algorithm 
and reference solution is about $0.41 \%$.

Numerical Example 3

Consider an infinite viscoelastic plate containing a hole of unit radius, subjected to an internal uniform pressure of unity. Plane strain conditions are assumed with Young's Modulus $E_{1}=0.1 \mathrm{MPa}$, $E_{2}=0.1 \mathrm{MPa}, \eta_{1}=1 \times 10^{7} \mathrm{~N} \cdot \mathrm{s} / \mathrm{m}^{2}$ and Poisson ratio $v=0.3$.

Due to the biaxial symmetry of the problem, only one quarter of the plate is considered as shown in Fig. 11. If the centre of the hole is defined as the scaling centre, nodes only need to set along the boundary of the hole, and are arranged with a uniform $\Delta s=l / 6$ where $l$ is the length of the boundary.

The solution of $u_{x}$ at node $C$ given by the proposed algorithm with adaptive sizes of time steps is presented in Table 5 and Fig. 12, and is compared with a reference solution [37]. The maximum relative error between the proposed algorithm and reference solution is about $0.035 \%$.

\section{Numerical Example 4}

Consider an infinte plate with a through crack subjected uniform tension, as shown in Fig. 13 (a) where $a=1 \mathrm{~m}$ and $\sigma_{0}=1 \mathrm{~N} / \mathrm{m}$. Plane stress conditions are assumed with $E_{1}=2000 \mathrm{~Pa}, E_{2}=2000 \mathrm{~Pa}$, $\eta_{1}=8 \times 10^{5} \mathrm{~N} \cdot \mathrm{s} / \mathrm{m}^{2}, v=0.25$.

Due to the biaxial symmetry, only one quarter of the plate is considered as shown in Fig. 13 (b). where the computing model of EFG-SBM consists of a bounded domain with the scaling centre at the crack tip, and an unbounded domain with the scaling centre at the middle of the crack.

The solution of $u_{x}$ at node $A$ given by the proposed algorithm with adaptive sizes of time steps is presented in Table 6 and Fig. 14, and compared with a reference solution given by [11, 38]. The maximum relative error between the proposed algorithm and reference solution is about $0.9 \%$.

\section{Computing remarks}

(1) In the section 3, a consecutive 3 checks based principle is presented to secure the computing accuracy. Actually numerical test of example 2 indicates that only one check is sufficient.

(2) For examples 1, 3, and 4, the elastic solutions related to the reference solutions can be obtained analytically, it means that these reference solutions are exact solutions. However example 2 needs a FEM based convergent elastic solution that requires more DOF of unknowns in comparison with EFG-SBM. In this example, the proposed algorithm needs only 50 DOF, the convergent elastic solution given by ANSYS needs 30618 DOF. An ANSYS based viscoelastic analysis for this problem may lead to more DOF.

(3) Fig 8 exhibits the adaptive procedure of size of time interval. When creep tends to a stable state, the variation of displacement becomes less and less, and the size of time step gradually becomes relative bigger in the adaptive process. When the size of time step is enlarged, more recursive computing may be required to satisfy Eqn (76) as shown in Figs 9.

\section{Conclusions}

This paper proposes an EFG-SBM based numerical method via a combination with a temporally adaptive algorithm for the viscoelastic analysis, which may be particularly beneficial to solve viscoealtic problems with unbounded domains or singularity. The work presented extends the 
application of SBM since there seems no any direct report relevant to SBM exploration on the viscoelastic problems. The major merits include

a) A spatially and temporally coupled viscoelastic problem is converted into a series of recurrent boundary value problems which are solved by EFG-SBM that is semi-analytical, and advantageous in dealing with the problems involving unbounded domains and singularity.

b) A self-adaptive computation can be realized in the time domain to adapt the change of the size of time step, and results in a more precise description for the variation of variables.

c) In the whole recursive process, the eigenvalue equations and the stiffness matrix need to be solved only one time.

d) Both creep and relaxation analyses can easily be carried out using the proposed algorithm.

\section{Acknowledgement}

The research leading to this paper is funded by NSF (11572068, 11202046), "973 Program" [2015CB057804].

\section{References}

[1] Wolf JP, Song Ch. Consistent infinitesimal finite element cell method: three dimensional vector wave equation. Int J Numer Methods Eng 1996;39:2189-2208.

[2] Wolf JP, Song Ch. Finite-Element Modelling of Unbounded Media. Chichester: John Wiley \& Sons; 1996.

[3] Wolf JP, Song Ch. The scaled boundary finite-element method - a primer: derivation. Comput Struct 2000;78:191-210.

[4] Wolf JP, Song Ch. The scaled boundary finite-element method - a fundamental solution-less boundary-element method. Comput Methods Appl Mech Eng 2001;190:5551-5568.

[5] Deeks AJ, Wolf JP. Semi-analytical elastostatic analysis of two-dimensional unbounded domains. Int J Numer Anal Methods Geomech 2002;26:1031-1057.

[6] Deeks AJ, Wolf JP. A virtual work derivation of the scaled boundary finite-element method for elastostatics. Comput Mech 2002;28:489-504.

[7] Deeks AJ, Augarde CE. A meshless local Petrov-Galerkin scaled boundary method. Comput Mech 2005;36:159-170.

[8] Yang HT, Guo XF, He YQ. An EFG-SBM based partitioning algorithm for heat transfer analysis of cyclically symmetrical structures. Finite Elem Anal Des 2015;93:42-49.

[9] Guo XF, He YQ, Yang HT. An EFG-SBM based partitioning algorithm for two-dimensional elastic analysis of cyclically symmetrical structures. Eng Computation 2015;32(2):452-472.

[10] Shames IH, Cozzarelli FA. Elasitc and inelastic stress analysis. Englewood Cliffs, New Jersey: Prentice Hall; 1992.

[11] Cai E. The foundation of viscoelastic mechanics. Beijing: Beihang University Press; 1989.

[12] Christensen RM. Theory of viscoelasticity: an introduction. New York: Academic Press; 1982. 
[13] Zhao WJ, Chen LQ, Zu JW. Finite difference method for simulating transverse vibrations of an axially moving viscoelastic string. Appl Math Mech (English Edition) 2006;27(1):23-28.

[14] Barbosa FS, Farage MCR. A finite element model for sandwich viscoelastic beams: Experimental and numerical assessment. J Sound Vib 2008;317:91-111.

[15] Chen H, Feng X, Huang Y, Huang YG, Rogers JA. Experiments and viscoelastic analysis of peel test with patterned strips for applications to transfer printing. J Mech Phys Solids 2013;61:1737-1752.

[16] Lima AMG, Guaraldo-Neto B, Sales TP, Rade DA. A time-domain modeling of systems containing viscoelastic materials and shape memory alloys as applied to the problem of vibration attenuation. Eng Struct 2014;68:85-95.

[17] Gong SW, Klaseboer E. Interaction between collapsing bubble and viscoelastic solid: Numerical modelling and simulation. Appl Math Model 2015;000:1-19.

[18] Sogabe Y, Nakano M et al. Finite element analysis of dynamic behaviour of viscoelastic materials using FFT. JSME Int J Series A-Mech Mater Eng 1996;39:71-77.

[19] Schmidt A, Gaul L. Finite element formulation of viscoelastic constitutive equations using fractional time derivatives. Nonlinear Dynam 2002;29:37-55.

[20] Leung A, Zhu B et al. Two-dimensional viscoelastic vibration by analytic Fourier P-elements. Thin-Walled Structures 2003;41:1159-1170.

[21] Sorrentino S, Fasana A. Finite element analysis of vibrating linear systems with fractional derivative viscoelastic models. J Sound Vib 2007;299:839-853.

[22] Kim SY, Lee DH. Identification of fractional-derivative-model parameters of viscoelastic materials from measured FRFs. J Sound Vib 2009;324:570-586.

[23] Han Z, Yang HT. A piecewise temporal expanding algorithm to solve viscoelastic problems with large deformation. Chinese Journal of Solid Mechanics 2013;34(4):380-387.

[24] Yang HT, Guo XL. Perturbation boundary-finite element combined method for solving the linear creep problem. Inter J Solids and Struct 2000;37:2167-2183.

[25] Katsikadelis JT, Babouskos NG. Post-buckling analysis of viscoelastic plates with fractional derivative models. Eng Anal Bound Elem 2010;34:1038-1048.

[26] León EP, Ochoa DS, Rodríguez-Castellanos A, Aliabadi MH, Gauna EA, Olivera-Villaseñor E. Creeping analysis with variable temperature applying the boundary element method. Eng Anal Bound Elem 2012;36:1715-1720.

[27] Yang HT, Liu Y. A combined approach of EFGM and precise algorithm in time domain solving viscoelasticity problems. Inter J Solids and Struct 2003;40:701-714.

[28] Han Z, Yang HT, Liu L. Solving viscoelastic problems with cyclic symmetry via a precise algorithm and EFGM. Acta Mech. Sinica 2006;22:170-176.

[29] Peng MJ, Li RX, Cheng YM. Analyzing three-dimensional viscoelasticity problems via the improved element-free Galerkin (IEFG) method. Eng Anal Bound Elem 2014;40:104-113.

[30] Gilhooley DF, Xiao JR, Batra RC, McCarthy MA, Gillespie Jr. JW. Two-dimensional stress 
analysis of functionally graded solids using the MLPG method with radial basis functions.

Comp Mater Sci 2008;41:467-481.

[31] Paola MD, Pirrotta A, Valenza A. Visco-elastic behavior through fractional calculus: An easier method for best fitting experimental results. Mech Mater 2011;43:799-806.

[32] Barbera D, Chen HF. Creep rupture assessment by a robust creep data interpolation using the Linear Matching Method. European Journal of Mechanics A/Solids 2015;54:267-279.

[33] Chen SS, Xu CJ, Tong GS. A meshless local natural neighbour interpolation method to modelling of functionally graded viscoelastic materials. Eng Anal Bound Elem 2015;52:92-98.

[34] Zienkiewicz OC, Morgan K. Finite Element and Approximation. New York: Wiley-Interscience Publication; 1983.

[35] Belytschko T, Lu YY, Gu L. Element-free Galerkin methods. Int J Numer Methods Eng 1994;37:229-256.

[36] Belytschko T, Krongauz Y, Organ D. Meshless methods: an overview and recent developments. Comput Methods Appl Mech Eng 1996;139:3-47.

[37] Wu JL. Elasticity. Beijing: Higher Education Press; 2001.

[38] Chen J, Zhao SS. Mechanics of fracture. Beijing: Science Press; 2006. 


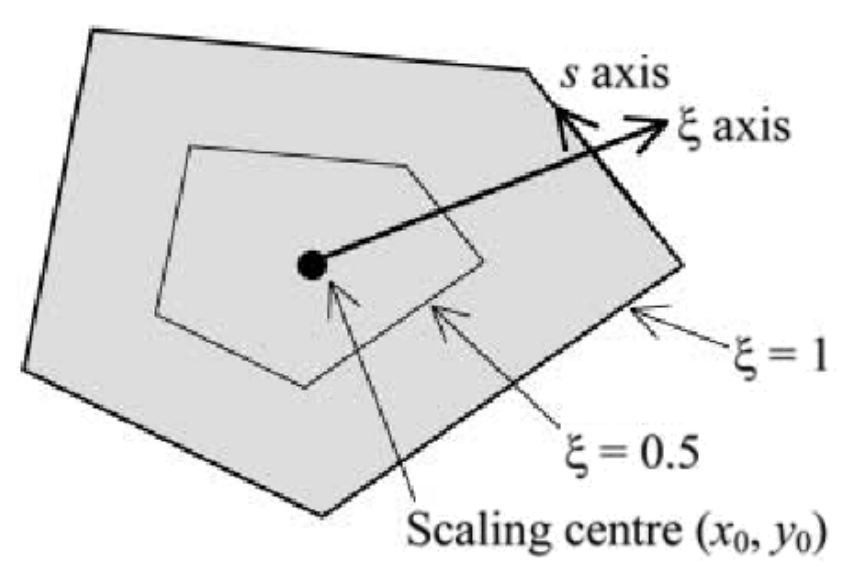

Fig. 2. Definition of the scaled boundary coordinate system. 


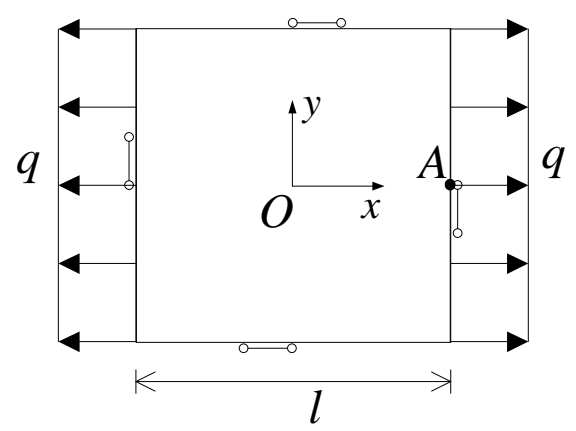

(a)

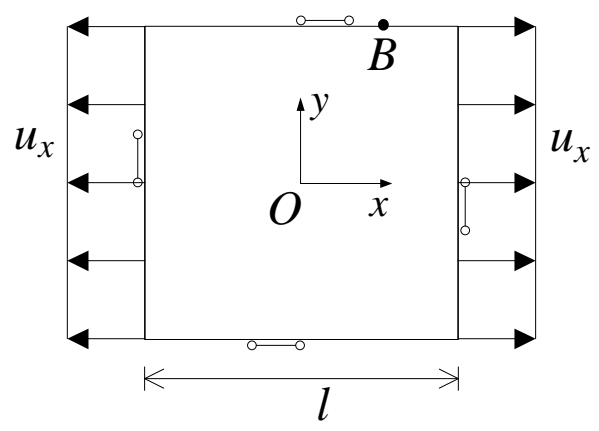

(b)

Fig. 3. A square plate subjected to uniform tension or prescribed displacement along two opposite sides ( $x_{A}=0.5 \mathrm{~m}, y_{A}=0$ and $\left.x_{B}=0.25 \mathrm{~m}, y_{B}=0.5 \mathrm{~m}\right)$. 


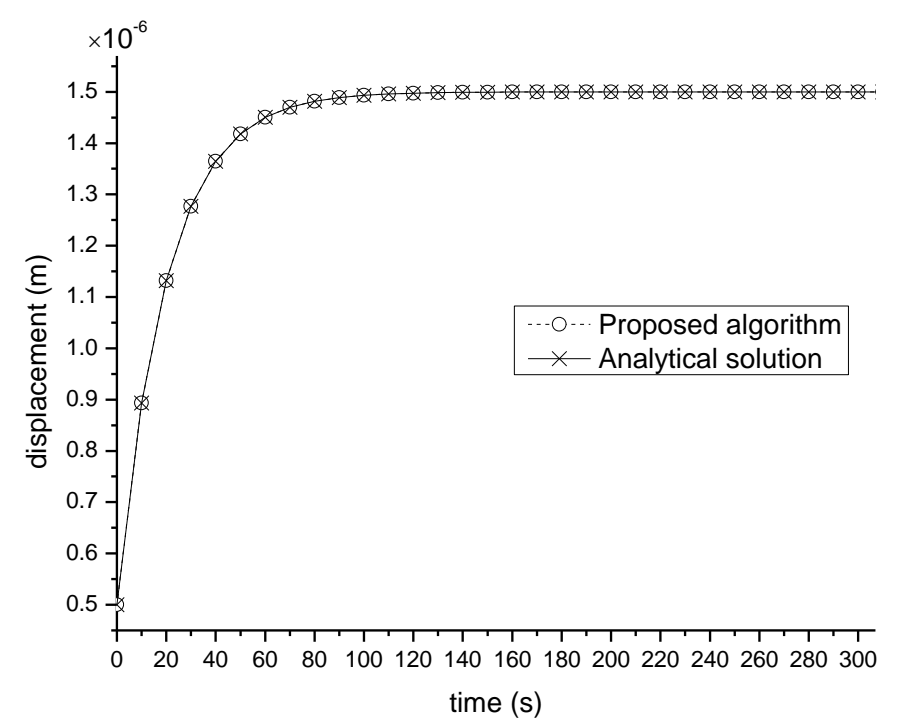

Fig. 4. Numerical comparison of $u_{x}$ at node $A$. 


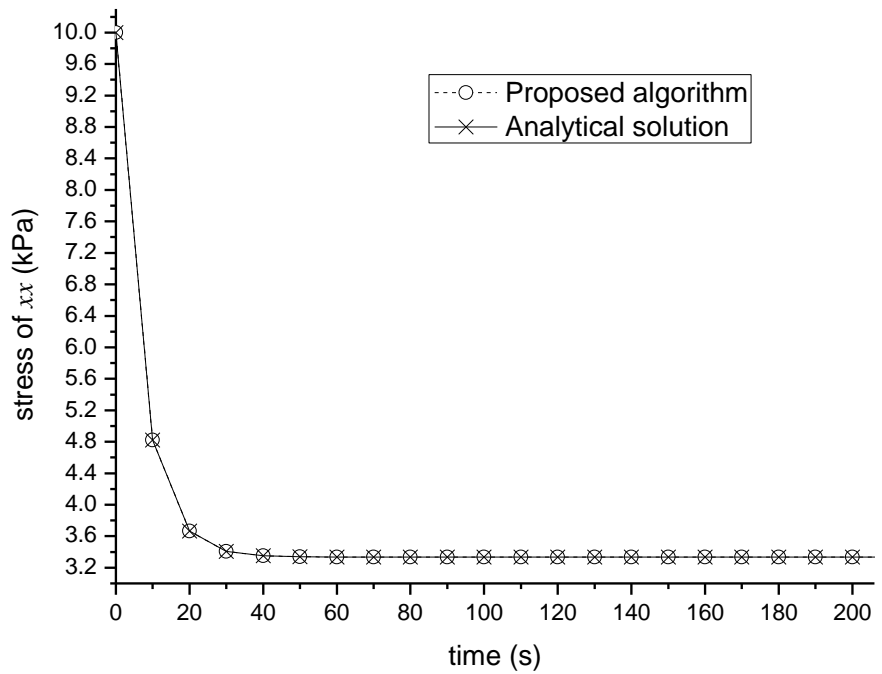

Fig. 5. Numerical comparison of $\sigma_{x x}$ at node $B$. 


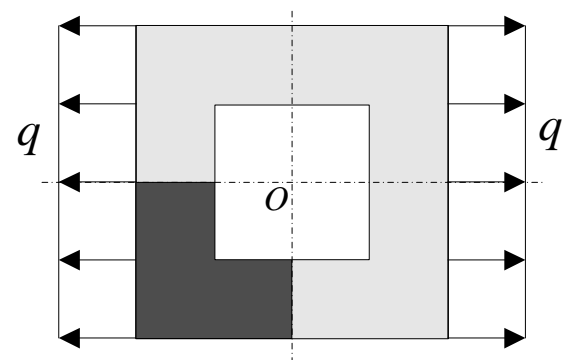

(a)

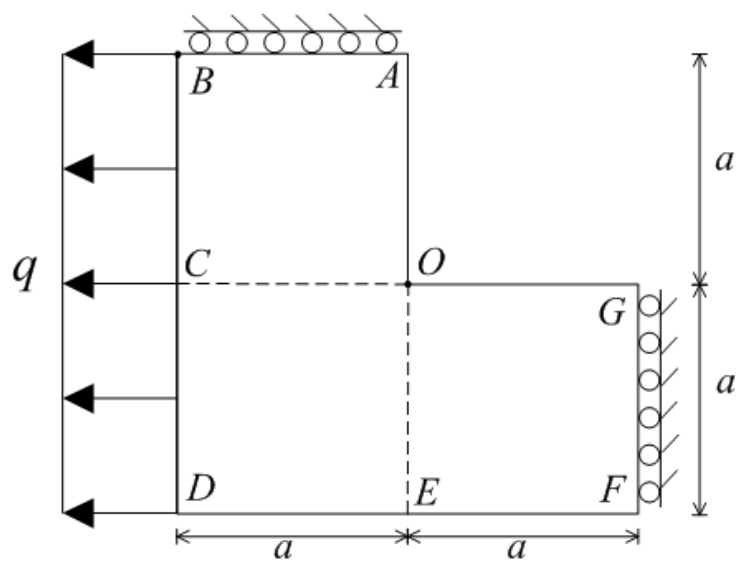

(c)

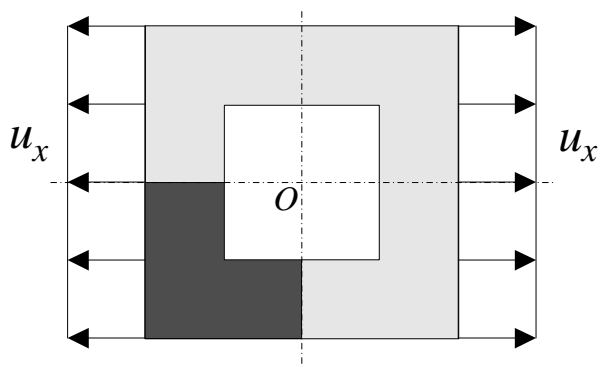

(b)

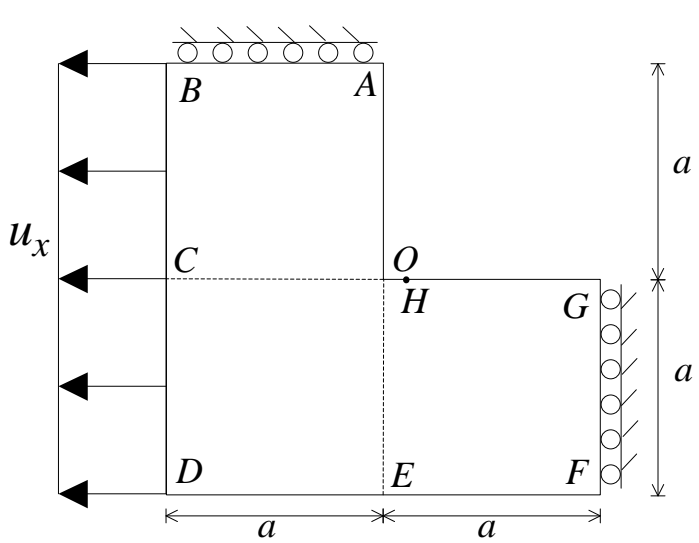

(d)

Fig. 6. A square plate with a square hole with uniform tension or prescribed displacement at two sides. 


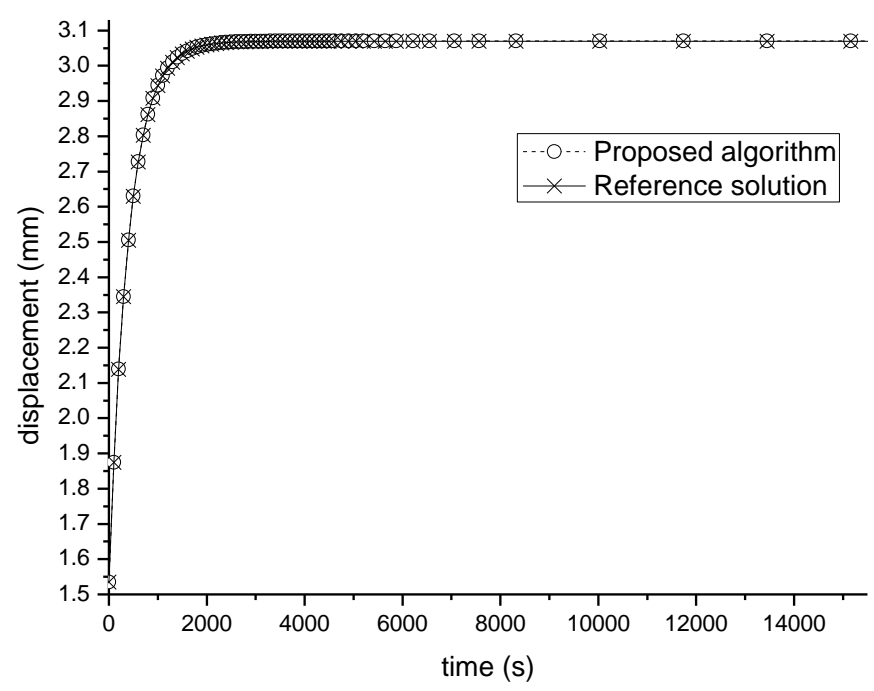

Fig. 7. Numerical comparison of $u_{y}$ at node $O$. 


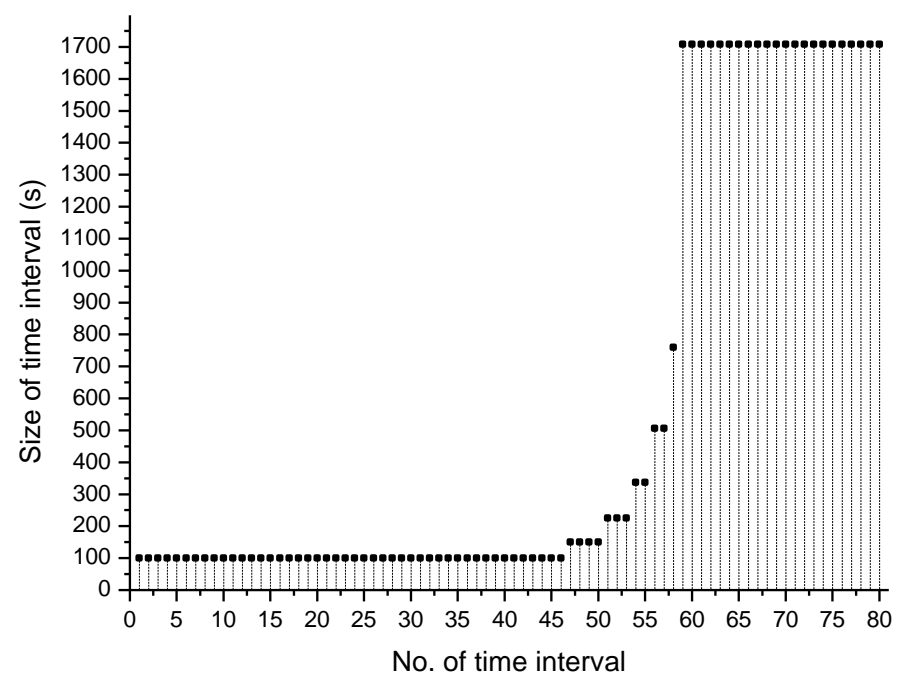

Fig. 8. Size of time interval. 


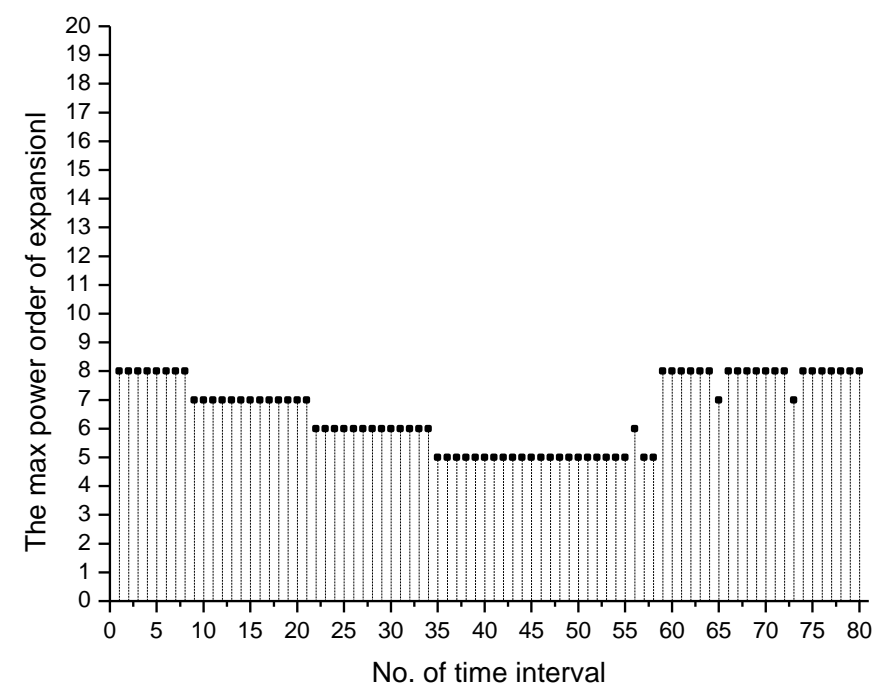

Fig. 9. The max power order of expansionl at each time interval. 


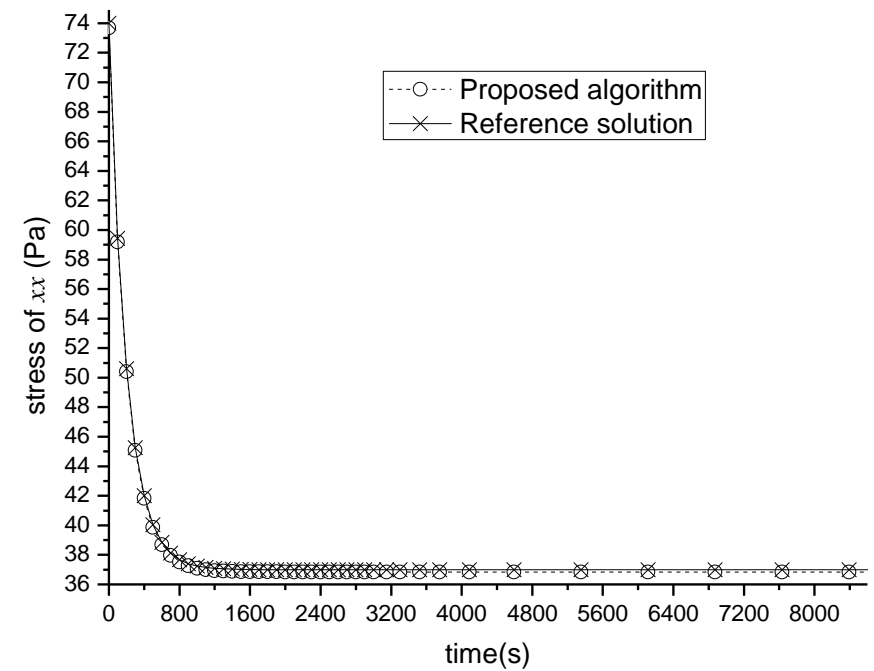

Fig. 10. Numerical comparison of $\sigma_{x x}$ at point $H$. 


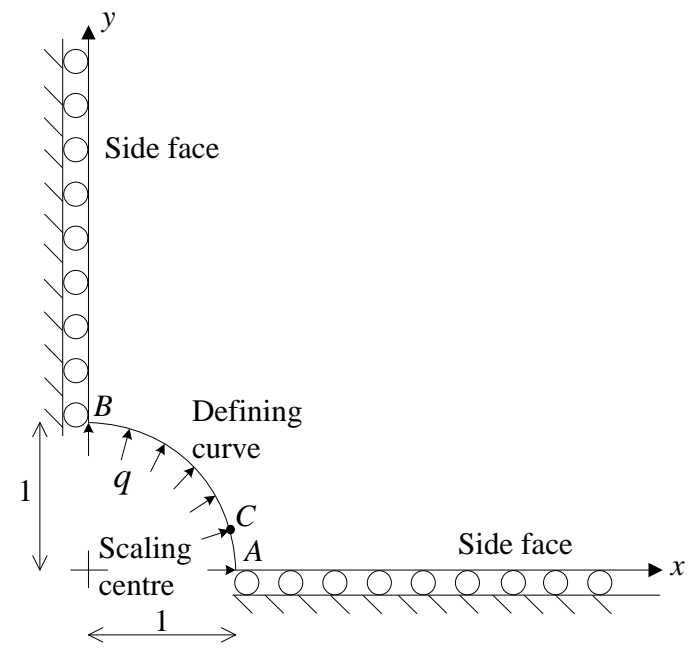

Fig. 11. Model for plane strain plate of infinite extent with circular hole subjected to an internal uniform pressure ( $\left.x_{C}=0.9659 \mathrm{~m}, y_{C}=0.2588 \mathrm{~m}\right)$. 


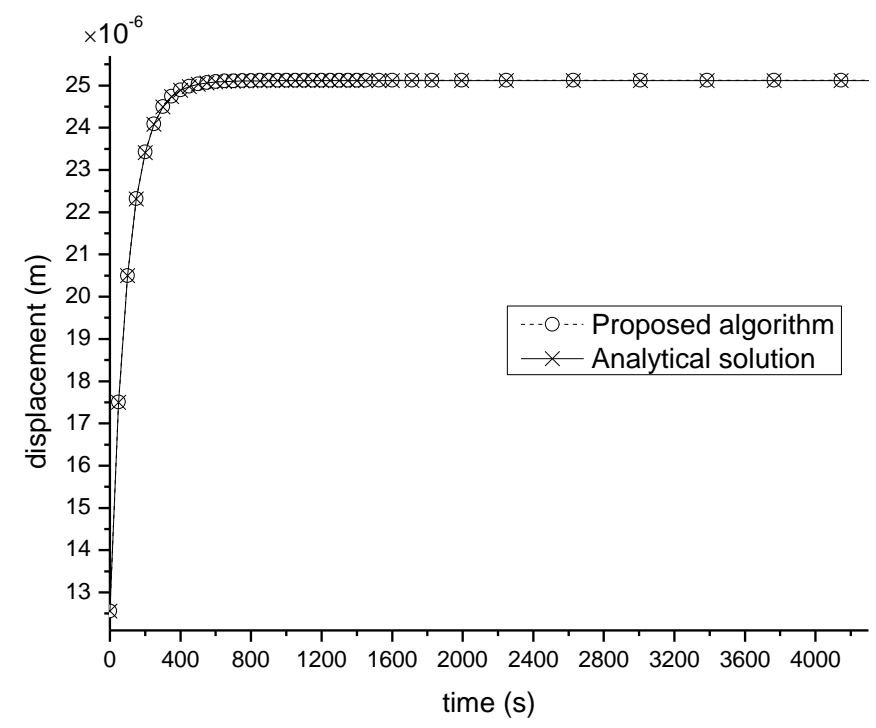

Fig. 12. Numerical comparison of $u_{x}$ at node $C$. 


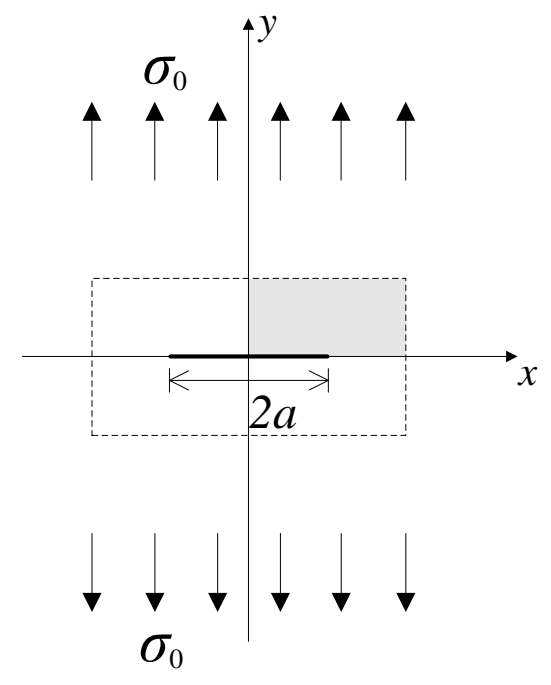

(a)

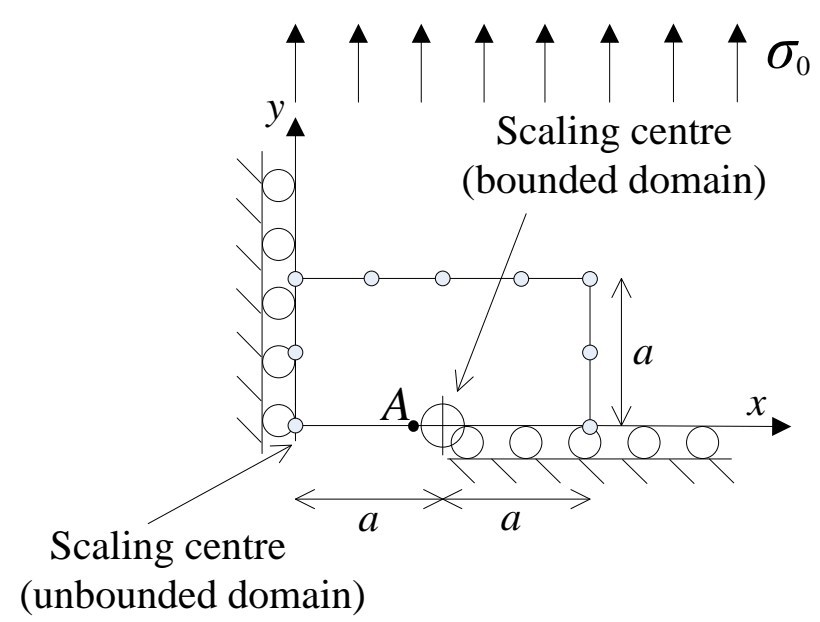

(b)

Fig. 13. An infinte plate with a through crack subjected uniform tension. 


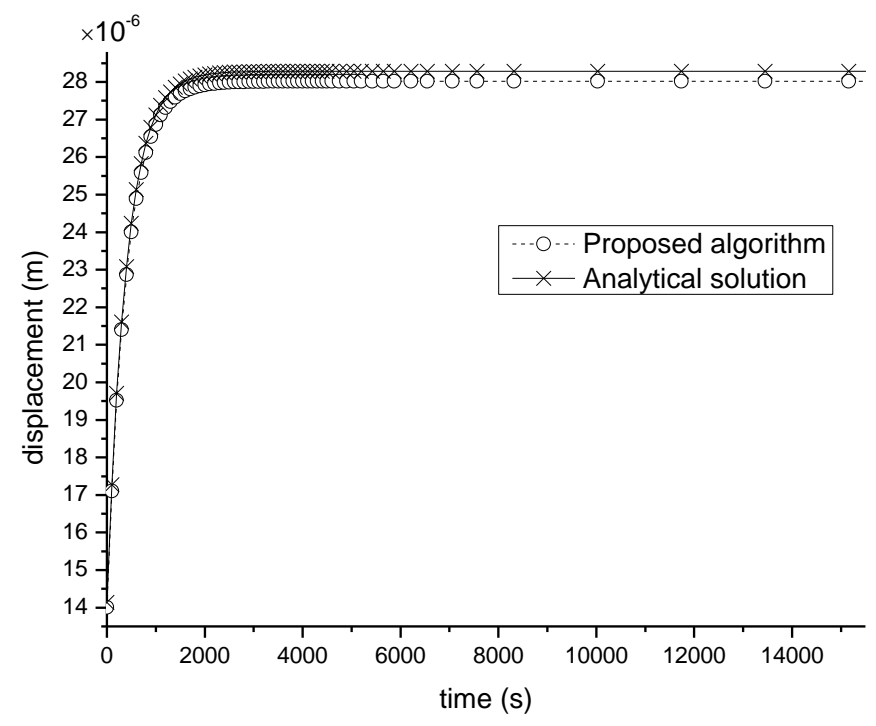

Fig. 14. Numerical comparison of $u_{y}$ at point $A$. 
Table 1 Numerical comparison of $u_{x}$ at node $A\left(x_{A}=0.5 \mathrm{~m}, y_{A}=0\right)$

\begin{tabular}{|c|c|c|}
\hline $\begin{array}{c}t \\
(\mathrm{~s})\end{array}$ & $\begin{array}{c}\text { Proposed } \\
\text { algorithm }\left(10^{-6} \mathrm{~m}\right)\end{array}$ & $\begin{array}{c}\text { Analytical } \\
\text { solution }\left(10^{-6} \mathrm{~m}\right)\end{array}$ \\
\hline 10 & 0.8934693 & 0.8934693 \\
\hline 20 & 1.1321206 & 1.1321206 \\
\hline 30 & 1.2768698 & 1.2768698 \\
\hline 40 & 1.3646647 & 1.3646647 \\
\hline 50 & 1.4179150 & 1.4179150 \\
\hline 60 & 1.4502129 & 1.4502129 \\
\hline 70 & 1.4698026 & 1.4698026 \\
\hline 80 & 1.4816844 & 1.4816844 \\
\hline 90 & 1.4888910 & 1.4888910 \\
\hline 100 & 1.4932621 & 1.4932621 \\
\hline 110 & 1.4959132 & 1.4959132 \\
\hline
\end{tabular}


Table 2 Numerical comparison of $\sigma_{x x}$ at node $B\left(x_{B}=0.25 \mathrm{~m}, y_{B}=0.5 \mathrm{~m}\right)$

\begin{tabular}{|c|c|c|}
\hline $\begin{array}{c}t \\
(\mathrm{~s})\end{array}$ & $\begin{array}{c}\text { Proposed } \\
\text { algorithm(kPa) }\end{array}$ & $\begin{array}{c}\text { Analytical } \\
\text { solution(kPa) }\end{array}$ \\
\hline 10 & 4.8208677 & 4.8208677 \\
\hline 20 & 3.6652471 & 3.6652471 \\
\hline 30 & 3.4073933 & 3.4073933 \\
\hline 40 & 3.3498583 & 3.3498583 \\
\hline 50 & 3.3370206 & 3.3370206 \\
\hline 60 & 3.3341561 & 3.3341561 \\
\hline 70 & 3.3335169 & 3.3335169 \\
\hline 80 & 3.3333743 & 3.3333743 \\
\hline 90 & 3.3333425 & 3.3333425 \\
\hline 100 & 3.3333354 & 3.3333354 \\
\hline 110 & 3.3333338 & 3.3333338 \\
\hline
\end{tabular}


Table 3 Numerical comparison of $u_{y}$ at point $O\left(x_{O}=1 \mathrm{~m}, y_{O}=1 \mathrm{~m}\right)$ after 1,2 and 3 checks

\begin{tabular}{|c|c|c|c|c|}
\hline \multirow{2}{*}{$\begin{array}{c}t \\
(\mathrm{~s})\end{array}$} & \multicolumn{3}{|c|}{ Proposed algorithm (mm) } & \multirow{2}{*}{$\begin{array}{l}\text { Reference } \\
\text { solution } \\
(\mathrm{mm})\end{array}$} \\
\hline & 1 check & 2 checks & 3 checks & \\
\hline 100.00 & 1.8747711 & 1.8747711 & 1.8747711 & 1.8741744 \\
\hline 200.00 & 2.1392382 & 2.1392382 & 2.1392382 & 2.1385574 \\
\hline 300.00 & 2.3452054 & 2.3452054 & 2.3452054 & 2.3444591 \\
\hline 400.00 & 2.5056128 & 2.5056128 & 2.5056128 & 2.5048154 \\
\hline 500.00 & 2.6305382 & 2.6305382 & 2.6305382 & 2.6297011 \\
\hline 600.00 & 2.7278303 & 2.7278303 & 2.7278303 & 2.7269621 \\
\hline 700.00 & 2.8036014 & 2.8036014 & 2.8036014 & 2.8027091 \\
\hline 800.00 & 2.8626119 & 2.8626119 & 2.8626119 & 2.8617009 \\
\hline 900.00 & 2.9085694 & 2.9085694 & 2.9085694 & 2.9076438 \\
\hline 1000.00 & 2.9443612 & 2.9443612 & 2.9443612 & 2.9434242 \\
\hline
\end{tabular}


Table 4 Numerical comparison of $\sigma_{x x}$ at point $H\left(x_{H}=1.1 \mathrm{~m}, y_{H}=1 \mathrm{~m}\right)$

\begin{tabular}{|c|c|c|}
\hline $\begin{array}{c}t \\
(\mathrm{~s})\end{array}$ & $\begin{array}{c}\text { Proposed } \\
\text { algorithm(Pa) }\end{array}$ & $\begin{array}{c}\text { Reference } \\
\text { solution(Pa) }\end{array}$ \\
\hline 100.00 & 59.1925326 & 59.4352083 \\
\hline 200.00 & 50.3994418 & 50.6060678 \\
\hline 300.00 & 45.0661626 & 45.2509234 \\
\hline 400.00 & 41.8313652 & 42.0028641 \\
\hline 500.00 & 39.8693615 & 40.0328166 \\
\hline 600.00 & 38.6793460 & 38.8379224 \\
\hline 700.00 & 37.9575652 & 38.1131824 \\
\hline 800.00 & 37.5197830 & 37.6736054 \\
\hline 900.00 & 37.2542546 & 37.4069884 \\
\hline 1000.00 & 37.0932035 & 37.2452771 \\
\hline
\end{tabular}


Table 5 Numerical comparison of $u_{x}$ at node $C\left(x_{C}=0.9659 \mathrm{~m}, y_{C}=0.2588 \mathrm{~m}\right)$

\begin{tabular}{|c|c|c|}
\hline $\begin{array}{c}t \\
(\mathrm{~s})\end{array}$ & $\begin{array}{c}\text { Proposed } \\
\text { algorithm }\left(10^{-6} \mathrm{~m}\right)\end{array}$ & $\begin{array}{c}\text { Analytical } \\
\text { solution }\left(10^{-6} \mathrm{~m}\right)\end{array}$ \\
\hline 50.00 & 17.504106 & 17.497844 \\
\hline 100.00 & 20.501931 & 20.494596 \\
\hline 150.00 & 22.320203 & 22.312218 \\
\hline 200.00 & 23.423041 & 23.414661 \\
\hline 250.00 & 24.091946 & 24.083327 \\
\hline 300.00 & 24.497657 & 24.488893 \\
\hline 350.00 & 24.743734 & 24.734882 \\
\hline 400.00 & 24.892987 & 24.884081 \\
\hline 450.00 & 24.983513 & 24.974575 \\
\hline 500.00 & 25.038420 & 25.029463 \\
\hline
\end{tabular}


Table 6 Numerical comparison of $u_{y}$ at point $A\left(x_{A}=0.9999 \mathrm{~m}, y_{A}=0\right)$

\begin{tabular}{|c|c|c|}
\hline$t$ & $\begin{array}{c}\text { Proposed } \\
\text { algorithm }\left(10^{-6} \mathrm{~m}\right)\end{array}$ & $\begin{array}{c}\text { Analytical } \\
\text { solution }\left(10^{-6} \mathrm{~m}\right)\end{array}$ \\
\hline 100.00 & 17.107717 & 17.270365 \\
\hline 200.00 & 19.521041 & 19.706632 \\
\hline 300.00 & 21.400539 & 21.603999 \\
\hline 400.00 & 22.864293 & 23.081670 \\
\hline 500.00 & 24.004266 & 24.232482 \\
\hline 600.00 & 24.892079 & 25.128734 \\
\hline 700.00 & 25.583507 & 25.826737 \\
\hline 800.00 & 26.121992 & 26.370341 \\
\hline 900.00 & 26.541365 & 26.793701 \\
\hline 1000.00 & 26.867973 & 27.123414 \\
\hline
\end{tabular}

